\title{
The Significance of the Entropic Linkage in the Theory of Measurements
}

\author{
Vladimír MaJerník \\ Institute of Physics, Slovak Academy of Sciences, \\ Bratislava 9, Czechoslovakia \\ Received October 31, 1968
}

\begin{abstract}
This paper deals with the mathematical description of the statistical linkage between the probability systems from point of view of measurement theory. It is shown that a suitable measure for the statistical linkage represents the amount of information contained in the random variable defined on the pointer positions of measuring instrument about the measured random variable defined on the set of physical states of measured object. The information becomes so a physical quantity that expresses the magnitude of so-called entropic linkage between the physical probability systems representing, in frame of entropic model of measurement, the measured object and measuring instrument. Physical aspects and the mathematical properties of the measure for the entropic linkage between the measured object and measuring instrument are treated in this paper. To illustrate an application of described formalism, a calculation is presented in which the magnitude of Gaussian entropic linkage between the measured microobject and measuring instrument is determined.
\end{abstract}

\section{Introduction}

In the probability description of physical phenomena new sort of properties of physical statistical systems has been introduced in the physics. These properties are mainly connected with the internal structure of statistical systems, e.g. with the order, the set-up or organisation of its elements. In frame of mathematical description of these properties certain quantitative measures, adequate to the physical and mathematical requirements, were found. Entropy, playing an important rôle in the thermodynamics and statistical physics, belongs to the most important measure for a property of said sort. Precise mathematical definition of the term "entropy" showns that the general entropy can be explicitly determined through the elements of the probability distribution of a random variable defined on a physical probability system. Therefore, the entropy of a random variable may be an adequate measure of its probability uncertainty [1], a concept being introduced and studied in frame of information theory [2]. The connection between the general entropy and physical entropy was found by $J_{A Y N E S}$ in his principle of the "maximum entropy estimation" well known in the statistical mechanics [3]. 
Between the probability systems a statistical linkage may exist which is mathematically given by a set of values of the conditional probabilities for the occurrence of the elements of first system dependent on the elements of second one. A measure for the statistical linkage was at first introduced in information theory. Its mane is the information contained in a probability system about the other one [4].

Mathematical formalism of information theory may be successfully applied in the description of the physical probability systems as well. Between the element set-up in the first and in the second physical probability systems a certain coherence exists which forms a new entropic property. This may be called the entropic linkage between these systems. It was shown in the information theory that an adequate scalar measure of this entropic linkage is even the information. Similarly as the entropy the information also acquires a character of a physical quantity which enables one to describe an ensemble of physical probability systems with statistical linkages.

Formalism used by description of two physical probability systems with entropic linkage has an immediate application in the mathematical description of the entropic aspect in the theory of measurements. If one considers the measuring process as a process by which on the side of measuring instrument the information about the measured object is gained, then he comes, at a great extent of abstraction, to so-called entropic model of measurement [5]. The main features of this model may be summerized as follows:

(i) the measured object and measuring instrument are considered as two physical probability systems,

(ii) on the set of physical states of the measured object, the measured parameter is given as a random variable $\tilde{f}_{1}$ with a probability distribution,

(iii) on the set of pointer positions of the measuring instrument is given a random variable $\tilde{f}_{2}$, too,

(iv) during the measuring process between the random variables $\tilde{f}_{1}$ and $f_{2}$ a statistical linkage is established, the measure of which is given by the information contained in the random variable $f_{2}$ about the random variable $\tilde{f}_{1}$.

In the next sections, using the entropic model of measurement, we shall deal with the mathematical treatment of the physical probability systems, the measured object and measuring instrument. As a concrete application of the developed formalism, the magnitude of the entropic linkage between the measured microobject with the measured random variable being one from a couple of the complementary physical quantities, will be calculated. From point of view of the theory of measurements, we are able to observe only the probability system representing the measuring instrument being linked with measured object by an entropic 
linkage. The larger the magnitude of this entropic linkage is the more information on measured object may be gained by a measuring process.

\section{Mathematical Model}

Let us consider a probability system consisting of the elements $x_{1}^{\prime}, x_{2}^{\prime}, \ldots, x_{n}^{\prime}$ from the set $X^{\prime}$. Let the class of the subsets

$$
\mathscr{X}^{\prime}=\left[E_{1}^{\prime}, E_{2}^{\prime}, \ldots, E_{n}^{\prime}\right]
$$

of set $X^{\prime}$ forms a $\sigma$-algebra on which a probability measure $P_{X}$ is given. Finally, let $\beta_{1}=f_{1}\left(x^{\prime}\right)$ be a $\mathscr{X}^{\prime}$-measurable function on the set $X^{\prime}$ having its values from the set $X$. By the tetrad $\left\{X^{\prime}, \mathscr{X}^{\prime}, P_{X}, \beta_{1}\right\}$ containing the elements of a probability field together with the random variable $\beta_{1}$, is a probability system $\Sigma_{1}$ mathematically described. This probability system may be understood either in the information theory as an information source $Q_{1}$ or in physics as a general physical probability system $\Sigma_{f}^{(1)}$.

Let us further consider another probability system $\Sigma_{2}$ mathematically described by the tetrad $\left\{Y^{\prime}, \mathscr{Y}^{\prime}, P_{Y}, \beta_{2}\right\}$ containing a probability field with the random variable $\beta_{2}$ defined on the elements $y_{1}^{\prime}, y_{2}^{\prime}, \ldots, y_{n}^{\prime}$ of the set $Y^{\prime}$, the values of which belong to the set $Y$. This system may be considered either as a receiver of information $Q_{2}$ or as a general physical probability system $\Sigma_{f}^{(2)}$ : Between the random variable $\beta_{1} \in \Sigma_{1}$ and $\beta_{2} \in \Sigma_{2}$ generally exists a statistical linkage determined for each point $x \in X$ by a probability measure $r_{x}$ defined on the $\sigma$-algebra $\mathscr{Y}=\left[F_{1}, F_{2}, \ldots, F_{i}\right], F_{k}$ denotes a subset of set $Y$, which, as a functions of $x$, is $\mathscr{X}$-measurable $\left(\mathscr{X}=\left[E_{1}, E_{2}, \ldots, E_{j}\right], E_{i}\right.$ denotes a subset of set $X)$. The triad of data $C=\left\{\mathscr{X}, r_{x}, \mathscr{Y}\right\}$ represents mathematical abstraction for the communication channel and, in physics, the entropic linkage between the physical probability systems.

The scalar measure for the statistical linkage between the random variable $\beta_{1} \in \Sigma_{1}$ and the random variable $\beta_{2} \in \Sigma_{2}$ is defined by so-called average information $I\left(\beta_{1}, \beta_{2}\right)$ contained in random variable $\beta_{2}$ about the random variable $\beta_{1}$. The information is generally given by a LebesgueStieltjes integral

$$
I\left(\beta_{1}, \beta_{\mathbf{2}}\right)=\int_{X \otimes Y} \log f(x, y) P_{X Y}(d x d y),
$$

where $P_{X Y}$ is the joint probability distribution given on the set of ordered couples $(x, y), x \in X, y \in Y$, and $f(x, y)$ is the density of information defined by the expression [6]

$$
f(x, y)=\frac{P_{X Y}(d x d y)}{P_{X}(d x) P_{Y}(d y)} .
$$

The quantity $I\left(\beta_{1}, \beta_{2}\right)$ gives in terms of information theory the amount of information about the random variable $\beta_{1} \in Q_{1}$ contained in the 
random variable $\beta_{2} \in Q_{2}$. In Physics it gives the magnitude of the entropic linkage between the physical probability systems $\Sigma_{f}^{(1)}$ and $\Sigma_{f}^{(2)}$.

In the case of discrete probability distribution of the random variable $\beta_{1}$,

$$
\mathscr{P}=\left[P_{1}, P_{2}, \ldots, P_{i}, \ldots, P_{n}\right],
$$

and a transfer matrix $R$ [7] determined by a set of the conditional probabilities, $r_{i}(j)$, for occurrence of $j$-th value of the random variable $\beta_{2}$, if the random variable $\beta_{1}$ assumes its $i$-th value, the expression for information (1) is turned out to be

$$
I\left(\beta_{1}, \beta_{2}\right)=\sum_{i, j} P_{i} r_{i}(j) \log \frac{r_{i}(j)}{\sum_{k} P_{k} r_{k}(j)} .
$$

In the case of an absolutely continuous distribution function of random variable $\beta_{1}$, the probability distribution (2) may be characterised also by a function of the probability density $p(x)$. For the transfer matrix we obtain a transfer function $r_{x}(y)$ determined by the density function of conditional probability of the value $y$ of the random variable $\beta_{2}$, when the random variable $\beta_{1}$ assumes the value $x$. The information in this case is given by a Riemann integral

$$
I\left(\beta_{1}, \beta_{2}\right)=\int_{X} \int_{Y} p(x) r_{x}(y) \log \frac{r_{x}(y)}{q(y)} d x d y,
$$

where

$$
q(y)=\int_{X} p(x) r_{x}(y) d x
$$

\section{The Gaussian Entropic Linkage Between the Complementary Systems}

In physics often emarges the problem of the physical realisations of probability systems, as well as, on the other hand, that of the reduction of physical probability systems on their mathematical probability models. Of importance is looking for a suitable physical realisations of the general probability systems in the theory of measurements and information. In both cases the mathematical probability systems with a large information content are requested which by its physical realisation leads to the requirement of the maximal entropic linkage between the corresponded physical probability systems. But a large entropic linkage may, due to the physical interaction connected with it, on the other hand, causes a change of the original probability distribution of the systems $\Sigma_{f}^{(1)}$ and $\Sigma_{f}^{(2)}$. This is, however, highly unfavourable at physical measurements. Ideal would be the couple of systems $\Sigma_{f}^{(1)}$ and $\Sigma_{f}^{(2)}$ that have the maximum value of entropic linkage by the minimum magnitude of physical interaction. How closely may these requirements be satisfied is a fundamental problem which may be solved by considering the general lows of quantum and statistical physics. 
We now turn to the determination of entropic linkage between two physical probability systems, the measured microobject $\left(\Sigma_{f_{1}}^{(1)}\right)$ and measuring instrument $\left(\Sigma_{f_{2}}^{(2)}\right)$, when on the measured microobject a physical quantity, being from a couple of complementary quantities $F$ and $G$, is to be measured. The quantity $F$ gets on the systems $\Sigma_{f_{1}}^{(1)}$ and $\Sigma_{f_{2}}^{(2)}$ the form of the random variables $f_{1} \in \Sigma_{f_{1}}^{(1)}$ and $\tilde{f}_{2} \in \Sigma_{f_{2}}^{(2)}$. Let $x$ or $y$ be the actual value of $f_{1}$ or $f_{2}$, respectively. During the measurement a statistical linkage between the random variables $f_{1}$ and $f_{2}$ is established. When this statistical linkage is a deterministrical one, i.e.

$$
r_{x}(y)=\delta(x-y)
$$

then the measuring instrument shows the same value of measured variable as it is on the measured object. This would represent the ideal case of measurement. The type of statistical linkage differs generally from the deterministical one being dependent, due to the uncertainty relations, on the value of dispersion of the complementary quantity $G$ given on the measuring instrument. In what follows we shall determine the magnitude of entropic linkage between the measured microobject and measuring instrument, when measuring a physical quantity $F$ by given value of the dispersion of quantity $G$ on the measuring instrument. For the sake of simplicity, a couple of introduced systems shall be called the complementary systems.

We shall consider the complementary physical systems $\Sigma_{f_{1}}^{(1)}$ and $\Sigma_{f_{2}}^{(2)}$ on which the random variables $\tilde{f}_{1}$ and $\tilde{f}_{2}$ defined. Its probability distribution are given by the density functions $p(x)$ and $q\left(y, \sigma_{\tau}\right)$, where $\sigma_{\tau}$ is the dispersion of the complementary quantity $G$ at the measuring instrument. According to the uncertainty relation, for the product of the dispersions of the random variable $\tilde{f}_{2}$, denoted by $\sigma_{y}$, and that of its complementary quantity, $\sigma_{\tau}$, we have [8]

$$
\sigma_{y} \sigma_{\tau} \geqq \frac{h}{4 \pi} \text {. }
$$

Taking the optimal case in the inequality (5), we get

$$
\sigma_{y} \sigma_{\tau}=\frac{h}{4 \pi},
$$

where the conditional density function (the transfer function) between the systems $\Sigma_{f_{1}}^{(1)}$ and $\Sigma_{f_{2}}^{(2)}$ is

$$
\begin{aligned}
r_{x}(y) & =\frac{1}{\sqrt{2 \pi} \sigma_{y}} \exp \left\{-\frac{(y-x)^{2}}{2 \sigma_{y}^{2}}\right\} \\
& =\frac{2 \sigma_{\tau}}{\sqrt{2 \pi} \hbar} \exp \left\{-\frac{2(y-x)^{2} \sigma_{\tau}^{2}}{\hbar^{2}}\right\} .
\end{aligned}
$$

By Eq. (7) the statistical linkage of Gaussian type is mathematically given. If the measured variable $\tilde{f}_{1}$ has the probability distribution $p(x)$, 
then due to Eq. (7) the corresponded distribution of the random variable $\tilde{f}_{2}$ on to pointer positions of the measuring instrument can be calculated, that may be in principle determined by a physical measurement, when $\sigma_{\tau}$ is given. The value of dispersion $\sigma_{\tau}$ is in a close connection with the physical parameters of the measuring process (e.g. with its duration).

Having the transfer function (7), the magnitude of entropic linkage may be calculated for every density function $p(x)$. The larger the magnitude of this entropic linkage is the more information about the measured parameter contains the measuring instrument. When the magnitude of entropic linkage between the complementary systems is zero, then both probability systems are stochastically independent. In this case the value on measuring instrument does not say anything about the value of the measured parameter. Therefore the system $\Sigma_{f_{2}}^{(2)}$ cannot do the function of a measuring instrument. In terms of information theory it means that between the systems $\Sigma_{f_{1}}^{(1)}$ and $\Sigma_{f_{2}}^{(2)}$ no information may be transmitted. Since the uncertainty relations have a universal validity it is in principle impossible, under such conditions, to realize physically the information transfer.

We now turn to calculation of the magnitude of Gaussian entropic linkage $I\left(f_{1}, f_{2}\right)$. Using Eqs. (7) and (4), we get for the information in dependence on the dispersion

$$
\begin{aligned}
I\left(\sigma_{\tau}\right)= & \frac{1}{\sqrt{2 \pi}} \int_{X} \int_{Y} p(x) \frac{2 \sigma_{\tau}}{\hbar} \exp \left\{-\frac{(y-x)^{2} 2 \sigma_{\tau}^{2}}{\hbar^{2}}\right\} \\
& \cdot \log \left[\frac{\frac{2 \sigma_{\tau}}{\sqrt{2 \pi} \hbar} \exp \left\{-\frac{(y-x)^{2} 2 \sigma_{\tau}^{2}}{\hbar^{2}}\right\}}{q(y)}\right] d x d y
\end{aligned}
$$

where

$$
q(y)=\frac{1}{\sqrt{2} \bar{\pi}} \int_{X} p(x) \frac{2 \sigma_{\tau}}{\hbar} \exp \left\{-\frac{(y-x)^{2} 2 \sigma_{\tau}^{2}}{\hbar^{2}}\right\} d x .
$$

If $p(x)$ is given, then it is possible to determine the magnitude of the Gaussian entropic linkage between the systems $\Sigma_{f_{1}}^{(1)}$ and $\Sigma_{f_{2}}^{(2)}$ for each $p(x)$. The probability distribution of the random variable $f_{2}$ on the measuring instrument, $q(y)$, is connected with the probability distribution of the measured variable, $p(x)$, by an integral transformation (9) with the Gaussian kernel. Since [9]

$$
\delta(y-x)=\lim _{\sigma_{\tau} \rightarrow \infty} \frac{2 \sigma_{\tau}}{\sqrt{2 \pi} \hbar} \exp \left\{-\frac{(y-x)^{2} 2 \sigma_{\tau}^{2}}{\hbar^{2}}\right\},
$$

we get $q(y)=p(x)$, when $\sigma_{\tau} \rightarrow \infty$, i.e. in this case the probability distributions on the system $\Sigma_{f_{1}}^{(1)}$ and $\Sigma_{f_{2}}^{(2)}$ are identical. Next, the value of $I\left(f_{1}, \tilde{f}_{2}\right)$ for certain types of the probability distribution of $f_{1}$ will be calculated. 


\section{The Magnitude of the Entropic Linkage Between the Complementary Systems}

The problems connected with the determination of the magnitude of entropic linkage between the systems $\Sigma_{f_{1}}^{(1)}$ and $\Sigma_{f_{2}}^{(2)}$ may be divided up into three classes:

1. To find such a probability distribution $p(x)$ for which the magnitude of entropic linkage between the systems becomes extreme.

2 . To find the probability distribution $p(x)$ for which the magnitude of entropic linkage gets its extreme value under cetrain boundary condition laid on this distribution.

3. To find the magnitude of entropic linkage, when a concrete probability distribution, $p(x)$, is given.

Since the first two classes of problems belong to a group of highly complicated variation problems, we shall confine ourselves to the problem of third one. We shall therefore look for the magnitude of entropic linkage of the probability distribution given by the following density functions:

(i) $p_{1}(x)=\left(\delta\left(x_{1}-x\right)+\delta\left(x_{2}-x\right)\right)$, i.e. the random variable assumes only two discrete values $x_{1}$ and $x_{2}$ with the probability $1 / 2$.

(ii) $p_{2}(x)=1 / Z=$ const. $x \in\langle 0, Z\rangle$, i.e. the probability distribution is within the interval $\langle 0, Z\rangle$ uniform.

(iii) $p_{3}(x)=a \cdot \exp \{-a x\}$, i.e. an exponential probability distribution. When $x$ denotes the energy, then by the density function $p_{3}(x)$ is represented the energy distribution low in an ideal gas at the thermodynamical equilibrium.

In order to illustrate the skeched formalism of the determination of entropic linkage on a physical parameter of measurement, the results will be discussed for the case that the physical random variable $\tilde{f}_{1}$ is the energy having the complementary variable the time $T$. Taking for $\sigma_{\tau}$ the duration of measurement $T$ between the systems $\Sigma_{f_{1}}^{(1)}$ and $\Sigma_{f_{2}}^{(2)}$, the probability distribution of the variable $\tilde{f}_{2}$ turns out to be a time function. During the measuring process is the measuring instrument at the time $T$ in interaction with measured system $\Sigma_{f_{1}}^{(1)}$ therefore, an entropic linkage between both systems is established. From communication point of view it is desirable that the random variable $\tilde{f}_{2}$ should contain as much information as possible about the energy as the measured variable. Physically, this endeavour is characterized by the requirement of maximum entropic linkage between the systems $\Sigma_{f_{1}}^{(1)}$ and $\Sigma_{f_{2}}^{(2)}$. How far this requirement with regard of the measurement duration may be fulfilled we shall next see at some given probability distributions.

Let us now determine the magnitude of Gaussian entropic linkage between the systems $\Sigma_{f_{1}}^{(1)}$ and $\Sigma_{f_{2}}^{(2)}$, when the probability distribution of 17 Commun.math. Phys., Vol. 12 
$f_{1}$ is given by the density function

$$
p_{1}(x)=\frac{1}{2} \delta\left(x_{1}-x\right)+\frac{1}{2} \delta\left(x_{2}-x\right) .
$$

Inserting Eq. (11) into Eq. (8), we get

$$
\begin{aligned}
I\left(\sigma_{\tau}\right)= & \frac{1}{2 \sqrt{2 \pi}} \int_{X} \int_{Y}\left(\delta\left(x_{1}-x\right)+\delta\left(x_{2}-x\right)\right) \frac{2 \sigma_{\tau}}{\hbar} \exp \left\{-\frac{(y-x)^{2} 2 \sigma_{\tau}^{2}}{\hbar^{2}}\right\} \\
& \cdot \log \left[\frac{\frac{2 \sigma_{\tau}}{\sqrt{2 \pi} \hbar} \exp \left\{-\frac{(y-x)^{2} 2 \sigma_{\tau}^{2}}{\hbar^{2}}\right\}}{q(y)}\right] d x d y
\end{aligned}
$$

with

$$
q(y)=\frac{\sigma_{\tau}}{\sqrt{2 \pi} \hbar} \int_{X}\left(\delta\left(x_{1}-x\right)+\delta\left(x_{2}-x\right)\right) \exp \left\{-\frac{(y-x)^{2} 2 \sigma_{\tau}^{2}}{\hbar^{2}}\right\} d x .
$$

In expression (12) the integration with respect to the variable $x$ and partly to the variable $y(y \in\langle-\infty, \infty\rangle)$ leads to

where

$$
I\left(\sigma_{\tau}\right)=\log \frac{2 \sigma_{\tau}}{e \cdot \sqrt{2 \pi} \hbar}-J_{1}-J_{2},
$$

$$
\begin{aligned}
J_{1}= & \frac{\sigma_{\tau}}{\sqrt{2 \pi} \hbar} \int_{Y} \exp \left\{-\frac{\left(y-x_{1}\right)^{2} 2 \sigma_{\tau}^{2}}{\hbar^{2}}\right\} \\
& \cdot \log \left[\frac{\sigma_{\tau}}{\sqrt{2 \pi} \hbar}\left(\exp \left\{-\frac{\left(y-x_{1}\right)^{2} 2 \sigma_{\tau}^{2}}{\hbar^{2}}\right\}+\exp \left\{-\frac{\left(y-x_{2}\right)^{2} 2 \sigma_{\tau}^{2}}{\hbar^{2}}\right\}\right)\right] d y
\end{aligned}
$$

and

$$
\begin{aligned}
J_{2}= & \frac{\sigma_{\tau}}{\sqrt{2 \pi} \hbar} \int_{Y} \exp \left\{-\frac{\left(y-x_{2}\right)^{2} 2 \sigma_{\tau}^{2}}{\hbar^{2}}\right\} \\
& \cdot \log \left[\frac{\sigma_{v}}{\sqrt{2 \pi} \hbar}\left(\exp \left\{-\frac{\left(y-x_{1}\right)^{2} 2 \sigma_{\tau}^{2}}{\hbar^{2}}\right\}+\exp \left\{-\frac{\left(y-x_{2}\right)^{2} 2 \sigma_{\tau}^{2}}{\hbar^{2}}\right\}\right)\right] d y .
\end{aligned}
$$

Since the integrals $J_{1}$ and $J_{2}$ are difficult to evaluate, we shall try to estimate only its certain limit values. When $x_{1}=x_{2}$, we get $I\left(\sigma_{\tau}\right)=0$. Assuming $x_{1} \ll x_{2}$, then in the integral $J_{1}$ or $J_{2}$ the term

$$
\exp \left\{-\frac{\left(y-x_{2}\right)^{2} 2 \sigma_{\tau}^{2}}{\hbar^{2}}\right\} \text { or } \exp \left\{-\frac{\left(y-x_{1}\right)^{2} 2 \sigma_{\tau}^{2}}{\hbar^{2}}\right\},
$$

respectively, may be neglected. Therefore we get $I\left(\sigma_{\tau}\right)=\log 2$. Since all terms within the integrals $J_{1}$ and $J_{2}$ are positive, we have

$$
J_{1} \geqq \frac{\sigma_{\tau}}{\sqrt{2 \pi} \hbar} \int_{Y} \exp \left\{-\frac{\left(y-x_{1}\right)^{2} 2 \sigma_{\tau}^{2}}{\hbar^{2}}\right\} \log \left[\frac{\sigma_{\tau}}{\sqrt{2 \pi} \hbar} \exp \left\{-\frac{\left(y-x_{1}\right)^{2} 2 \sigma_{\tau}^{2}}{\hbar^{2}}\right\}\right] d y
$$


and

$J_{2} \geqq \frac{\sigma_{\tau}}{\sqrt{2 \pi} \hbar} \int_{Y} \exp \left\{-\frac{\left(y-x_{2}\right)^{2} 2 \sigma_{\tau}^{2}}{\hbar^{2}}\right\} \log \left[\frac{\sigma_{\tau}}{\sqrt{2 \pi} \hbar} \exp \left\{-\frac{\left(y-x_{2}\right)^{2} 2 \sigma_{\tau}^{2}}{\hbar^{2}}\right\}\right] d y$

Taking into account the inequalities (13a) and (13b) one can determine the limits of the expression $I\left(\sigma_{\tau}\right)$ for $\Delta z=x_{2}-x_{1} \in\langle 0, \infty\rangle$. If $\sigma_{\tau}<\infty$, then

$$
0 \leqq I\left(\sigma_{\tau}\right) \leqq \log 2 .
$$

From the form of integrals $J_{1}$ and $J_{2}$ it can be seen that the more the value of $I\left(\sigma_{\tau}\right)$ approaches to zero or to $\log 2$, the smaller or greater, respectively, $\sigma_{\tau}$ and $\Delta z$ become. When $\sigma_{\tau} \rightarrow \infty$, we obtain

and

$$
\delta\left(x_{1}-y\right)=\lim _{\sigma_{\tau} \rightarrow \infty} \frac{2 \sigma_{\tau}}{\sqrt{2 \pi} \hbar} \exp \left\{-\frac{\left(y-x_{1}\right)^{2} 2 \sigma_{\tau}^{2}}{\hbar^{2}}\right\}
$$

$$
\delta\left(x_{2}-y\right)=\lim _{\sigma_{\tau} \rightarrow \infty} \frac{2 \sigma_{\tau}}{\sqrt{2 \pi} \hbar} \exp \left\{-\frac{\left(y-x_{2}\right)^{2} 2 \sigma_{\tau}^{2}}{\hbar^{2}}\right\},
$$

i.e. the probability distribution of random variable $\tilde{f}_{2}$ is identical with that of the random variable $\tilde{f}_{1}$. In this case the value of entropic linkage gets its maximum being equal to $\log 2$. When $\sigma_{\tau}=0$, we get for Eq. (12)

$$
\lim _{\sigma_{\tau} \rightarrow 0} I\left(\sigma_{\tau}\right)=0 \text {. }
$$

If $\Delta z<\infty$, then we have again

$$
0 \leqq I\left(\sigma_{\tau}\right) \leqq \log 2 .
$$

So it can be generally said that $I\left(\sigma_{\tau}\right)$, if $\sigma_{\tau}$ and $\Delta z \in\langle 0, \infty\rangle$, satisfies the inequality

$$
0 \leqq I\left(\sigma_{\tau}\right) \leqq \log 2
$$

Taking for $\sigma_{\tau}$ the duration of an energy measurement $T$, it means that for $T=0$ no entropic linkage between the systems $\Sigma_{f_{1}}^{(1)}$ and $\Sigma_{f_{2}}^{(2)}$ exists and that these are stochastically independent. No one of systems contains any information about the other one.

We shall next deal with the probability distribution given by density function of type:

$$
\begin{aligned}
& p_{2}(x)=1 / Z=\text { const. }, \quad x \in\langle 0, Z\rangle, \\
& p_{2}(x)=0, \quad x \in(Z, \infty\rangle .
\end{aligned}
$$

Substituing Eq. (14) into Eq. (8), we get

$$
\begin{aligned}
I\left(\sigma_{\tau}\right)= & \frac{2 \sigma_{\tau}}{\sqrt{2 \pi} \hbar} \int_{X} \int_{Y} \frac{1}{Z}-\exp \left\{-\frac{(y-x)^{2} 2 \sigma_{\tau}^{2}}{\hbar^{2}}\right\} \\
& \cdot \log \left[\frac{\frac{2 \sigma_{\tau}}{\sqrt{2 \pi} \hbar} \exp \left\{\frac{(y-x)^{2} 2 \sigma_{\tau}^{2}}{\hbar^{2}}\right\}}{q(y)}\right] d x d y
\end{aligned}
$$


with

$$
\begin{aligned}
q(y) & =\frac{2 \sigma_{\tau}}{\sqrt{2 \pi} \hbar} \int_{0}^{Z} \frac{1}{Z} \exp \left\{-\frac{(y-x)^{2} 2 \sigma_{\tau}^{2}}{\hbar^{2}}\right\} d x \\
& =\frac{1}{Z}\left(\Phi\left(\frac{(Z-y) 2 \sigma_{\tau}}{\hbar}\right)-\Phi\left(-\frac{2 y \sigma_{\tau}}{\hbar}\right)\right),
\end{aligned}
$$

where $\Phi(t)$ being the value of error integral for the argument $t$. The expression (15) can be written as the sum of three integrals

where

$$
I\left(\sigma_{\tau}\right)=J_{1}+J_{2}+J_{3}
$$

$$
\begin{aligned}
J_{1}= & \frac{2 \sigma_{\tau}}{\sqrt{2 \pi} \hbar} \int_{0}^{Z} \int_{-\infty}^{\infty} \frac{1}{Z} \exp \left\{-\frac{(y-x)^{2} 2 \sigma_{\tau}^{2}}{\hbar^{2}}\right\} \log \left(\frac{2 Z \sigma_{\tau}}{\sqrt{2 \pi} \hbar}\right) d x d y=\log \frac{2 Z \sigma_{\tau}}{\sqrt{2 \pi} \hbar}, \\
J_{2}= & \frac{2 \sigma_{\tau}}{\sqrt{2 \pi} \hbar} \int_{0}^{Z} \int_{-\infty}^{\infty} \frac{1}{Z} \exp \left\{-\frac{(y-x)^{2} 2 \sigma_{\tau}^{2}}{\hbar^{2}}\right\}\left(\frac{(y-x)^{2} 2 \sigma_{\tau}^{2}}{\hbar^{2}}\right) d x d y=1 / 2, \\
J_{3}= & \frac{2 \sigma_{\tau}}{\sqrt{2 \pi} \hbar} \int_{0}^{Z} \int_{-\infty}^{\infty} \frac{1}{Z} \exp \left\{-\frac{(y-x)^{2} 2 \sigma_{\tau}^{2}}{\hbar^{2}}\right\} \\
& \cdot \log \left[\Phi\left(\frac{(z-y) 2 \sigma_{\tau}}{\hbar}\right)-\Phi\left(-\frac{2 y \sigma_{\tau}}{\hbar}\right)\right] d x d y .
\end{aligned}
$$

Since these integrals are generally very complicated, we shall looking only for its approximative values under the assumption that $Z$ is sufficiently small. In this case the following approximative equation is held

$$
\frac{1}{Z}\left[\Phi\left(\frac{(Z-y) 2 \sigma_{\tau}}{\hbar}\right)-\Phi\left(-\frac{2 y \sigma_{\tau}}{\hbar}\right)\right] \approx \frac{2 \sigma_{\tau}}{\sqrt{2 \pi} \hbar} \exp \left\{-\frac{2 y^{2} \sigma_{\tau}^{2}}{\hbar^{2}}\right\} .
$$

Taking into account Eq. (16), we get

$$
\begin{aligned}
J_{3} \approx & -\frac{2 \sigma_{\tau}}{\sqrt{2 \pi} \hbar} \int_{0}^{Z} \int_{-\infty}^{\infty} \frac{1}{Z} \exp \left\{\frac{(y-x)^{2} 2 \sigma_{\tau}^{2}}{\hbar^{2}}\right\} \log \left(\frac{2 Z \sigma_{\tau}}{\sqrt{2 \pi} \hbar}\right) d x d y \\
& +\frac{2 \sigma_{\tau}}{\sqrt{2 \pi} \hbar} \int_{0}^{Z} \int_{-\infty}^{\infty} \frac{1}{Z} \exp \left\{-\frac{(y-x)^{2} 2 \sigma_{\tau}^{2}}{\hbar^{2}}\right\}\left(\frac{2 y^{2} \sigma_{\tau}^{2}}{\hbar^{2}}\right) d y d x \\
= & -\log \frac{2 Z \sigma_{\tau}}{\sqrt{2 \pi} \hbar}+1 / 2+\frac{2 Z^{2} \sigma_{\tau}^{2}}{3 \hbar^{2}} .
\end{aligned}
$$

Substituing Eq. (18) into Eq. (16), we can determine the approximate value of entropic linkage,

$$
I\left(\sigma_{\tau}\right) \approx \frac{2}{3}\left(\frac{Z \sigma_{\tau}}{\hbar}\right)^{2}
$$


From Eq. (19) it can be seen that for $\sigma_{\tau} \rightarrow 0$, we have

$$
\lim _{\sigma_{\tau} \rightarrow 0} I\left(\sigma_{\tau}\right)=0 \text {. }
$$

When $\sigma_{\tau}$ represents the duration of energy measurement, it means that the magnitude of entropic linkage between the systems $\Sigma_{f_{1}}^{(1)}$ and $\Sigma_{f_{2}}^{(2)}$ is zero, when $T=0$, for $T>0$ its value increases by the second power of the duration of measurement $T$. In terms of information theory it shows that the information contained in system $\Sigma_{f_{2}}^{(2)}$ about the system $\Sigma_{f_{1}}^{(1)}$ increases simultaneously with the enlargement of the measurement duration.

Finally we shall calculate the entropic linkage for the density function

$$
p_{3}(x)=a \exp \{-a x\}, \quad x \in\langle 0, \infty\rangle .
$$

Substituing the expression (20) into Eq. (8), we get

with

$$
\begin{aligned}
I\left(\sigma_{\tau}\right)= & \frac{2 \sigma_{\tau} a}{\sqrt{2 \pi} \hbar} \int_{0-\infty}^{\infty} \int_{-\infty}^{\infty} \exp \left\{-a x-\frac{(y-x)^{2} 2 \sigma_{\tau}^{2}}{\hbar^{2}}\right\} \\
& \cdot \log \left[\frac{\frac{2 \sigma_{\tau}}{\sqrt{2 \pi} \hbar} \exp \left\{-\frac{(y-x)^{2} 2 \sigma_{v}^{2}}{\hbar^{2}}\right\}}{q(y)}\right] d y d x
\end{aligned}
$$

$$
\begin{aligned}
q(y) & =\frac{2 a \sigma_{\tau}}{\sqrt{2 \pi} \hbar} \int_{0}^{\infty} \exp \left\{-a x-\frac{(y-x)^{2} 2 \sigma_{\tau}^{2}}{\hbar^{2}}\right\} d x \\
& =a \cdot \exp \left\{-a y+\frac{a^{2} \hbar^{2}}{8 \sigma_{\tau}^{2}}\right\} \cdot\left[1-\Phi\left(\frac{\hbar^{2} a-4 \sigma_{\tau}^{2} y}{\sqrt{2} \sigma_{\tau}}\right)\right]
\end{aligned}
$$

The expression (21) can be written as the sum of the integrals

where

$$
I\left(\sigma_{\tau}\right)=J_{1}+J_{2}+J_{3}
$$

$$
\begin{aligned}
J_{1}= & \frac{2 a \sigma_{\tau}}{\sqrt{2 \pi}} \int_{0}^{\infty} \int_{-\infty}^{\infty} \exp \left\{-a x-\frac{(y-x)^{2} 2 \sigma_{\tau}^{2}}{\hbar^{2}}\right\} \log \left(\frac{2 \sigma_{\tau}}{\sqrt{2 \pi} \hbar a}\right) d x d y \\
= & \log \frac{2 \sigma_{\tau}}{\sqrt{2 \pi} \hbar a}, \\
J_{2}= & \frac{2 a \sigma_{\tau}}{\sqrt{2 \pi}} \int_{0}^{\infty} \int_{-\infty}^{\infty} \exp \left\{-a x-\frac{(y-x)^{2} 2 \sigma_{\tau}^{2}}{\hbar^{2}}\right\} \\
& \cdot\left(-\frac{(y-x)^{2} 2 \sigma_{\tau}^{2}}{\hbar^{2}}+a y-\frac{a^{2} \hbar^{2}}{8 \sigma_{\tau}^{2}}\right) d x d y=1 / 2-\frac{a^{2} \hbar^{2}}{8 \sigma_{\tau}^{2}}, \\
J_{3}= & \int a \cdot \exp \left\{-a x+\frac{a^{2} \hbar^{2}}{8 \sigma_{\tau}^{2}}\right\} \\
& \cdot\left(-\left[1-\Phi\left(\frac{\hbar^{2} a-4 \sigma_{\tau}^{2} y}{2 \sigma_{\tau}}\right)\right] \log \left[1-\Phi\left(\frac{\hbar^{2} a-4 \sigma_{\tau}^{2} y}{2 \sigma_{\tau}}\right)\right]\right) d y .
\end{aligned}
$$


Since the function, $-t \log t$, for $t<1$ is positive, the integral $J_{3}$ gets also a positive value. Due to the fact that the function

$$
L\left(\sigma_{\tau}\right)=1-\Phi\left(\frac{\hbar^{2} a-4 \sigma_{\tau}^{2} y}{\sqrt{2} \sigma_{\tau}}\right)
$$

for $\sigma_{\tau} \rightarrow \infty$ becomes a step function assuming only two values, zero or 1 , the integral $J_{3}$ turns out to be zero. When $\sigma_{\tau}<\infty$, then $J_{3}>0$. Denoting by $J\left(\sigma_{\tau}\right)$ the value of integral $J_{3}$ in dependence on the parameter $\sigma_{\tau}$, we can write the result

$$
I\left(\sigma_{\tau}\right)=\log \frac{2 \sigma_{\tau}}{\sqrt{2 \pi} \hbar a}+\frac{1}{2}-\frac{a^{2} \hbar^{2}}{8 \sigma_{\tau}^{2}}+J\left(\sigma_{\tau}\right),
$$

which for $\sigma_{\tau} \rightarrow 0$ adopts the form

$$
I\left(\sigma_{\tau}\right)=\log \frac{2 \sigma_{\tau}}{\sqrt{2 \pi \hbar a}}+\frac{1}{2} .
$$

It may be seen that the entropic linkage between the systems $\Sigma_{j_{1}}^{(1)}$ and $\Sigma_{f_{2}}^{(2)}$ increases with enlargement of $\sigma_{\tau}$ and gets its extreme value for $\sigma_{\tau} \rightarrow \infty$.

Let us now consider the probability distribution on the set of pointer positions of measuring instrument given by Eq. (21a). From Eq. (21a) we have for the mean value

$$
\begin{aligned}
\bar{a}= & \int_{-\infty}^{\infty} y \cdot q(y) d y=\exp \left\{\frac{a^{2} \hbar^{2}}{8 \sigma_{\tau}}\right\} \int_{-\infty}^{\infty} y \exp \{-a y\} \\
& \cdot\left(1-\Phi\left(\frac{\hbar^{2} a-4 \sigma_{\tau} y}{\sqrt{2}}\right)\right) d y .
\end{aligned}
$$

Due to the property of the function $L$, we have for $\sigma_{\tau} \gg 1$

$$
\bar{a} \approx \exp \left\{\frac{a^{2} \hbar^{2}}{8 \sigma_{\tau}^{2}}\right\} a,
$$

i.e. that the expectation value of measured random variable (observable) is by measuring instrument changes in dependence of $\sigma_{\tau}$. When $\sigma_{\tau} \equiv T$, it means that the expectation value for energy is allways change by an energy measurement except of the case when $T=\infty$.

The foregoing results may be summerized in three points:

(i) When $\sigma_{\tau} \neq \infty$, the probability distribution of the pointer positions on measuring instrument is generally different from that of the physical states on the measured object.

(ii) Since the amount of information in system $\Sigma_{f_{2}}^{(2)}$ about the system $\Sigma_{f_{1}}^{(1)}$ is zero for $\sigma_{\tau}=0$, are in this case the systems $\Sigma_{f_{1}}^{(1)}$ and $\Sigma_{f_{2}}^{(2)}$ stochastically independent. Therefore, the system $\Sigma_{f_{2}}^{(2)}$ cannot serve as a measuring instrument. 
(iii) The magnitude of Gaussian entropic linkage between the measured microobject and measuring instrument assumes zero or maximum value, when $\sigma_{\tau}=0$ or $\infty$, respectively. For $\sigma_{\tau} \in(0, \infty), I\left(\tilde{f}_{1}, f_{2}\right)$ appears as a function of $\sigma_{\tau}$.

Finally it may be stated that the determination of the magnitude of entropic linkage between the measured microobject and measuring instrument is of fundamental importance in theory of measurements since it enables one to describe the measuring process as a process of information gaining on the side of an obsever. The treated Gaussian entropic linkage represents, however, only relatively small class of actual entropic linkages between the measured microobjects and measuring instrument, playing, nevertheless, an important rôle in the quantum theory of measurements.

\section{References}

1. Chintschin, A. J.: Der Begriff der Entropie in der Wahrscheinlichkeitsrechnung. Arbeiten zur Informationstheorie I. Berlin: VEB Deutscher Verlag d. Wissenschaften 1957.

2. Shannon, C. A.: BSTJ 27, 379-423, 623-657 (1948).

3. Jaynes, E. T.: Phys. Rev. 106, 620 (1957); 108, 106 (1957). - Katz, A.: Principle of statistical mechanics: The information-theoretical approach. San Francisco: Freeman 1967.

4. Billingsley, P.: Ergodic theory and information. New York: Wiley 1965.

5. MaJerník, V.: Entropic aspect of quantum theory of measurements. Thesis. Bratislava 1968.

6. Prochorov, Ju. V., Rozanov, Ju. A.: Probability theory (in Russian). p. 468. Moskva: Nauka 1967.

7. Frey, P.: Informationstheorie. p. 76. Berlin: Akademie-Verlag 1963.

8. Weinzet, W.: Lehrbuch der theoretischen Physik. S. 995. Berlin-GöttingenHeidelberg: Springer 1950.

9. Madelung, E.: Die mathematischen Hilfsmittel des Physikers. S. 18. BerlinGöttingen-Heidelberg: Springer 1950.

\author{
VLadimír MaJerníK \\ Institute of Physics \\ Slovak Academy of Sciences \\ Dúbravská cesta \\ Bratislava 9, ČSSR
}

